\title{
Biomechanics of ACL
}

\section{Modeling of the knee joint}

The function and biomechanics of the ACL can be understood only in conjunction with the entire knee joint which comprises three independent articulations, one between the patella and femur and the remaining two between the lateral and medial tibial and femoral condyles. ${ }^{1}$ To study the interaction of the cruciate ligaments with the tibiofemoral joint, a simplified two-dimensional, single degree-of-freedom "crossed four-bar linkage" moving in a single plane, is commonly used. ${ }^{2,3}$ Thus, the interaction between these four bars can be used to describe the motion of both the tibial and femoral condyles as well as the posterior migration of the tibiofemoral contact point that occurs with knee flexion. ${ }^{1,3}$ Because the model simplifies the degrees of freedom and reduces elongation of the cruciates during normal joint articulation, this approach may be inadequate for modeling more detailed interaction. Further mathematical and three dimensional models have been described. ${ }^{1,3}$

\section{Structural and mechanical properties of the ACL}

There have been a variety of testing conditions for evaluation of structural and mechanical properties of the ACL. ${ }^{4}$ Maturation and age of the specimen, ${ }^{5}$ methods of holding specimens, application of strain rates, ${ }^{5}$ angles of knee flexion, and the direction of the applied loads ${ }^{6}$ have important effects on the outcome of studies.

\section{Structural properties of the bone-ligament-bone complex}

The structural behavior of the bone-ligament-bone complex can be described by a load-deformation curve. The curve consists of different characteristic regions. Initially, little load is required to elongate the ligament. This is characterized by the relatively flat "toe" region of the curve. ${ }^{1}$

The change from the toe to the linear portion of the curve represents the change in stiffness that an examiner perceives during a clinical laxity examination when a ligament's endpoint is reached. ${ }^{1}$ The toe region is followed by a second, high stiffness region where significantly larger loads are required for continued elongation. Here, all the collagen fibers are straightened, and the curve becomes linear. This region characterizes the elastic deformation of the ligament. ${ }^{1}$

\section{Mechanical properties of the midsubstance ACL}

For determination of the mechanical properties of the midsubstance of the ligament itself, the cross-sectional area and change in length of the ligament must be determined. Measurements of the cross-sectional areas have been performed using contact (digital Vernier calipers, pressure area micrometers)and non-contact (shadow amplitude method, profile method, laser micrometer system). ${ }^{7}$ The approaches that have been developed to measure changes in length can also be categorized as contact or noncontact methods. Contact to the ligament was obtained by suturing mercuryfilled strain gauges onto ligaments ${ }^{8,9}$ or differential variable reluctance transducers ${ }^{11,12}$ to the ACL. Recently, noncontact methods such as high-speed camera systems, ${ }^{5,7}$ video dimension analyzers, or high-resolution video digitizing systems have been used. Time-dependence can be described by stress-relaxation and creep. ${ }^{12}$ Pioletti and Rakotomanana ${ }^{13}$ demonstrated that stress relaxation is important during the first seconds of a test, is moderate during the following minutes, and finally reaches a quasi-static state after 60 minutes.

\section{Impact of maturation and age}

The structural properties of the femur-ACL-tibia complex and the mechanical properties of the ligament mid substance are profoundly affected by maturation and age. Woo et al. ${ }^{5}$ demonstrated in rabbits, before and after closure of the epiphysis, that all specimens with open epiphyses failed by tibial avulsion, whereas those with closed epiphyses failed by tearing of the ligament substance.

\section{Length and isometry}

Early opinions were that an "isometric fiber" existed within the ACL; however, the "ideal" isometric attachment sites could not be found. When 
considering the entire ligament, the ACL isnot "isometric"1,14 The length of the PLB decreased by $1.5 \mathrm{~mm}(6 \%)$ and $7.1 \mathrm{~mm}(32 \%)$, respectively. Although isometric placement of ACL grafts is an ideal goal that cannot be achieved, it is possible to select attachment sites that minimize the change in tibiofemoral distances as the knee is flexed. ${ }^{3,}$ Furia et al ${ }^{14}$ suggest the concept of "physiologic isometry".

\section{Mechanical properties during passive motion}

Thus, when the leg is supported throughout flexion- extension motion, reconstructed ACL should be safe during continuous passive motion between full extension and $110^{\circ}$ of flexion..$^{1}$ If the knee is hyperextended or flexed over $120^{\circ}$, forces of more than $100 \mathrm{~N}$ and strains of up to $8 \%$ can be experienced. ${ }^{8}$

\section{Mechanical properties during muscle activity}

\subsection{Quadriceps}

In vivo studies demonstrated that there were positive strain levels on the AMB of more than $2 \%$ during isometric quadriceps contraction between full extension and $40^{\circ}$ of flexion. ${ }^{1,10}$ Bach and $\mathrm{Hull}^{8}$ showed similar results. The effect of quadriceps force application increased from no effect at $90^{\circ}$ and $120^{\circ}$ of flexion toward a mean increase of strain of $6 \%$ at full extension. Hamstrings

Bach and $\mathrm{Hull}^{8}$ showed that the simultaneous application of the hamstring force caused a reduction in strain relative to the quadriceps force alone.

\subsection{Mechanical properties under weigh bearing conditions}

Fleming et $\mathrm{al}^{12}$ implanted transducers in the intact ACL after arthroscopic meniscectomies. Immediately after surgery and while under local anesthesia, the knee was flexed to $20^{\circ}$ and attached to a six-degree of freedom toad cell. ACL strain was measured under non weight bearing and weight bearing conditions. increased strain within the ACL under compressive load have been attributed to two possible causes. ${ }^{12}$

\subsection{ACL as the primary restraint to anterior translation}

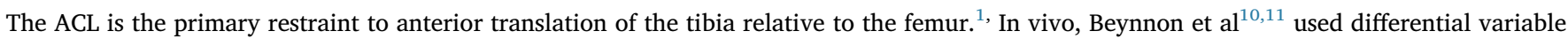
reluctance transducers and Hall-effect strain transducers which were attached by barbs to an intact AMB. For both the non weight bearing and weight bearing position at $30^{\circ}$ of flexion, they showed that an anterior load of more than $50 \mathrm{~N}$ produced ACL strains with increasing loads up to $6 \%$. Under non weight bearing conditions, cadaver studies demonstrated that the ACL provides an average restraint of $82 \%$ to $89 \%$ to the applied anteroposterior load at $30^{\circ}$ of flexion. This decreases slowly with increasing flexion to $74 \%$ to $85 \%$ at $90^{\circ}$ [1] . An increase of strain for the AMB and PLB was seen near extension and more than $90^{\circ}$ of flexion.

\subsection{ACL as a secondary restraint}

It has been consistently shown that the ACL is a secondary restraint to internal rotation in the non weight bearing and weight bearing knee, particularly when the joint is near extension. ${ }^{8,11,12}$ In vivo, Fleming et $\mathrm{al}^{12}$ recently demonstrated that the application of internal torques to the tibia between $0 \mathrm{Nm}$ and $10 \mathrm{Nm}$ produced strain in the ACL. In vivo, in the seated position and with the knee at $30^{\circ}$ of flexion, Beynnon et al ${ }^{11}$ demonstrated that the AMB was strained by $2 \mathrm{Nm}$ to $6 \mathrm{Nm}$ of internal torque. In cadaveric knee joints, the strain in both the AMB and PLB was found to be comparable between $15^{\circ}$ and $120^{\circ}$ of flexion. ${ }^{7}$ There is more disagreement about whether the ACL is a secondary restraint to external rotation and varus/valgus angulation. ${ }^{8,9,11}$ In vivo, Beynnon et $\mathrm{al}^{12}$ demonstrated that an external torque produced no strain in the AMB. In cadaveric knee joints, Bach and $\mathrm{Hull}^{8}$ could not find any strain in either bundle under an external torque. Bems et $\mathrm{al}^{9}$ showed that neither pure valgus torque, pure varus torque, nor pure rotation torque significantly strained the AMB at $30^{\circ}$ of flexion.

\section{CONCLUSION}

The ACL functions as a major primaryy restraint to internal rotation and as a minor secondary restraint to external rotation and varus-valgus angulation, particularly under weight-bearing conditions. Perfect understanding of biomechanics of ACL helps in great way in the management of ACL deficient knees.

\section{References}

1. Beynnon BD, Johnson RJ. Relevant biomechanics. In: DeLee JC, Drez D, eds. Orthopaedic-sports medicine. Philadelpia: WB Saunders; 1994:1113-1133.

2. Hefzy MS, Grood ES. Senailivily ol'in.scition locutions on length patterns of anterior cruciate ligament fibers. J Biomech Eng. 1986;108:73-82.

3. O'Connor J, ShercliffT, Fitzpatrick D, et al. Geometry of the knee. In: Daniel D, ed. Knee ligaments: structure, function, injury, and repair. New York: Raven Press; 1990:163-199.

4. Mueller W. Kinematics of the cruciate ligaments In Anonymous test. 1998:217-233.

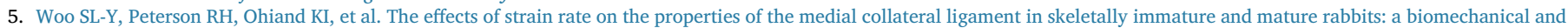
histological study. J Orthop Res. 1990;8:712-721.

6. Woo SL-Y, Hollis JM, Roux RD, et al. Effects of knee flexion on the structural properties of the rabbit femur anterior cruciate ligament - tibia complex (FATC). $J$ Biomech. 1987;20A:557-563.

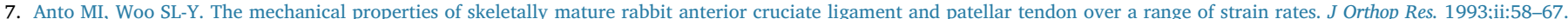

8. Bach JM, Hull ML. Strain inhoinogenily in the anterior cruciate ligament under application of external and muscular loads. J Biomech Eng. 1998;120:497-503.

9. Bems GS, Hull ML, Patterson HA. Strain in the anteromedial bundle of the anterior cruciate ligament under combination loading. $J$ Orthop Res. 1992;10:167-176.

10. Beynnon BD, Pope MH, Wertheimer CM, et al. The effect of functional knee-braces on strain on the anterior cruciate ligament in vivo. J Bone Joint Surg. 1992;74A:1298-1312.

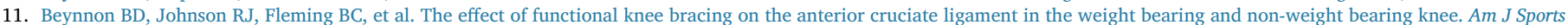
Med. 1997;25:353-359.

12. Fleming BD, Renstrom PA, Beynnon BD, et al. The effect of weight bearing and external loading on anterior cruciate ligament strain. $J$ Biomecli. 2001;34(163):70. 
13. Pioletti DP, Rakotomanana LR. On the interdependence of time and strain effects in the stress relaxation of ligaments and tendons. $J$ Biomech. $2000 ; 33: 1729-1732$.

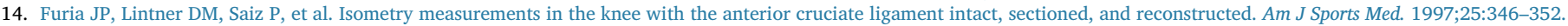

Professor of Orthopaedics P Gopinathan patinharayil, Professor dr. ${ }^{1}$ GMC Ortho Trust and Research Centre, CH cross road, Nadakkave, Calicut, kerala E-mail address: gopinathan.p@gmail.com

${ }^{1}$ Phone: 919447059014. 